\title{
Endoteliopatía corneal autoinmune como manifestación de uveitis intermedia
}

\author{
JORGE ORELLANA R. ${ }^{1}$, BASILIO ROJAS U. ${ }^{1}$, \\ FRANCISCO VILLARROEL C. ${ }^{1}$, PATRICIO ARÁNGUIZ Z. ${ }^{2}$ \\ 1. Fundación Oftalmológica Los Andes, Vitacura, Santiago. \\ 2. Unidad de Reumatología Infantil, Hospital Clínico San Borja-Arriarán, Servicio de Salud Metropolitano Central, Santiago.
}

\begin{abstract}
Corneal autoimmune endotheliopathy as manifestation of intermediate uveitis in children

Background: Intermediate Uveitis (IU) is an important cause of uveitis in children. It is considered a chronic intraocular inflammation that mainly affects the anterior vitreous and peripheral retina. Pars Planitis is a subtype of IU, consisting of a white opacity that covers the pars plana and ora serrata plus vitreous condensations in the eye. Corneal endothelium disease is a rare clinical finding associated with pars planitis. Objective: Describe the corneal manifestations in IU and its associated treatment between ophthalmology and pediatric rheumatology. Case-report: A 5 years-old boy with autoimmune endotheliopathy and unilateral pars planitis in the right eye. Initially, he was treated topically, but evolved with intraocular complications that required systemic medication. Long-term follow-up was performed, searching for associated systemic diseases, until one of these entities appeared. Conclusion: It is very important to search for inflammation in the anterior vitreous and pars plana in all children with corneal endotheliopathy, considering that an early and integral management of IU could diminish the risk of visual impairment as a complication.

(Key words: Intermediate uveitis, pars planitis, autoimmune endotheliopathy, children).

Rev Chil Pediatr 2008; 79 (5): 502-508
\end{abstract}

\section{RESUMEN}

Introducción: La Uveitis Intermedia (UI) es una causa importante de uveitis infantil. Se considera como una inflamación intraocular crónica que afecta principalmente a la retina periférica y vítreo anterior. La Pars Planitis es un subtipo de UI caracterizada por opacidades blanquecinas sobre la pars plana y ora serrata más condensaciones vítreas. La enfermedad del endotelio corneal es un hallazgo clínico infrecuente asociado con la Pars Planitis. Objetivo: Destacar la manifestación corneal de una UI y el tratamiento de la enfermedad entre oftalmólogo y reumatólogo infantil. Caso clínico: Se presenta caso clínico de niño de 5 años de edad con endoteliopatía autoinmune asociada a Pars Planitis unilateral del ojo derecho, que en principio se trató localmente, pero evolucionó con complicaciones intraoculares que requirieron medica-

Trabajo recibido el 26 de diciembre de 2007, devuelto para corregir el 11 de marzo de 2008, segunda versión el 13 de mayo de 2008, aceptado para publicación el 04 de junio de 2008. 
ción sistémica y se efectuó un seguimiento clínico por varios años buscando enfermedades sistémicas asociadas a UI hasta que ella apareció. Conclusión: Es necesario buscar la presencia de inflamación de pars plana y vítreo en todo niño que se presente con endoteliopatía corneal, considerando que el tratamiento oportuno y manejo integral del paciente con UI puede disminuir el riesgo de pérdida visual asociado a las complicaciones de esta enfermedad.

(Palabras clave: Uveitis intermedia, pars planitis, endoteliopatía autoinmune, niños).

Rev Chil Pediatr 2008; 79 (5): 502-508

\section{Introducción}

La uveitis intermedia (UI) es una entidad inflamatoria intraocular que compromete predominantemente a la retina periférica, vítreo anterior y pars plana.

Esta enfermedad afecta principalmente a pacientes bajo los 40 años y es responsable de entre un 8 a $22 \%$ de las causas de inflamación en población uveítica. El porcentaje de UI se incrementa en los menores de 16 años desde un 18 a $33 \%$ de todas las uveítis que los afectan. El pronóstico en adultos es usualmente favorable, pero en población pediátrica es incierto ${ }^{1}$.

El diagnóstico en niños es frecuentemente tardío, y puede presentarse con signos avanzados o complicaciones derivadas de su cronici$\mathrm{dad}^{2}$.

Algunos casos de remisión de la enfermedad han sido reportados, pero aún son imprecisos los datos relativos a la resolución de la UI en niños y los posibles cambios de la actividad inflamatoria en la adolescencia y adulte $z^{3}$.

Las manifestaciones clínicas de la UI en el segmento anterior del ojo son escasas, ya que generalmente ocurre en niños aparentemente saludables con un ojo poco sintomático. Aún así, puede haber ojo rojo, reacción de cámara anterior leve y otras manifestaciones infrecuentes como la aparición de opacidad corneal periférica a veces visible al examen externo, que puede ser secundario a edema corneal crónico, afección que se conoce como endoteliopatía autoinmune ${ }^{4,5}$.

En el segmento posterior del ojo es corriente encontrar opacidades vítreas de diversa intensidad semejantes a bolas de nieve o "snowballs", y depósitos de exudación inflamatoria que yacen sobre la base del vítreo, retina periférica y pars plana llamados bancos de nieve o "snowbanks".
A menudo se presenta vasculitis retinal, edema macular y, a veces, edema del disco óptico.

El término Pars Planitis (PP) ha sido usado para describir un subtipo específico de UI en el que existen "snowbanks" sobre pars plana y ora serrata, con mayor grado de inflamación y peor pronóstico visual que los pacientes con UI, cuyo origen es idiopático y su evolución es crónica $^{1,6}$.

Presentamos un caso clínico de PP unilateral que debutó con una manifestación clínica infrecuente en el segmento anterior del ojo, que fue tratado y controlado por largo tiempo por nosotros.

\section{Caso clínico}

Niño de 5 años, procedente de la comuna de Maipú, Santiago, que consultó en Septiembre del año 2000 por aparición de mancha blanca en su ojo derecho de 2 meses de evolución, según relato de su madre.

Fue evaluado en Centro de Referencia de Salud de Maipú y derivado al Servicio de Oftalmología del Hospital Clínico San Borja Arriarán.

Al examen oftalmológico presentaba una agudeza visual corregida de 0,5 en su ojo derecho y 1,0 en su ojo izquierdo. A la biomicroscopía, opacidad y engrosamiento corneal periférico y presencia de células inflamatorias en cámara anterior del ojo $($ Tyndall + ) sin enrojecimiento ocular externo. El examen de su ojo izquierdo resultó normal.

Fue catalogado como una endotelitis corneal de etiología desconocida y se decidió tratarlo con acetato de prednisolona al 1\% suspensión oftálmica desde 1 gota cada 2 horas hasta 1 gota día por medio, de acuerdo a la respuesta clínica y fue seguido regularmente hasta febrero 2001 . 
Su cuadro clínico cursó con remisiones y exacerbaciones de su inflamación ocular dependientes de la cantidad de corticoides tópicos administrados, hasta que en abril de 2001 se efectuó un minucioso examen de fondo de ojo derecho con oftalmoscopía binocular indirecta, encontrándose la presencia de un "snowbank", intenso sobre la pars plana inferior y "snowballs", flotando adyacentes en el vítreo inferior, diagnosticándose pars planitis y endoteliopatía autoinmune corneal en su ojo derecho. El ojo izquierdo no presentaba ninguna alteración en el segmento posterior (figuras 1 y 2).

El estudio ecográfico del ojo derecho demostró una separación parcial de la faz hialoídea posterior del cuerpo vítreo, sin tracción ni desprendimiento de retina asociado.

Se solicitó hemograma con recuento diferencial y velocidad de sedimentación globular los que resultaron normales y exámenes de

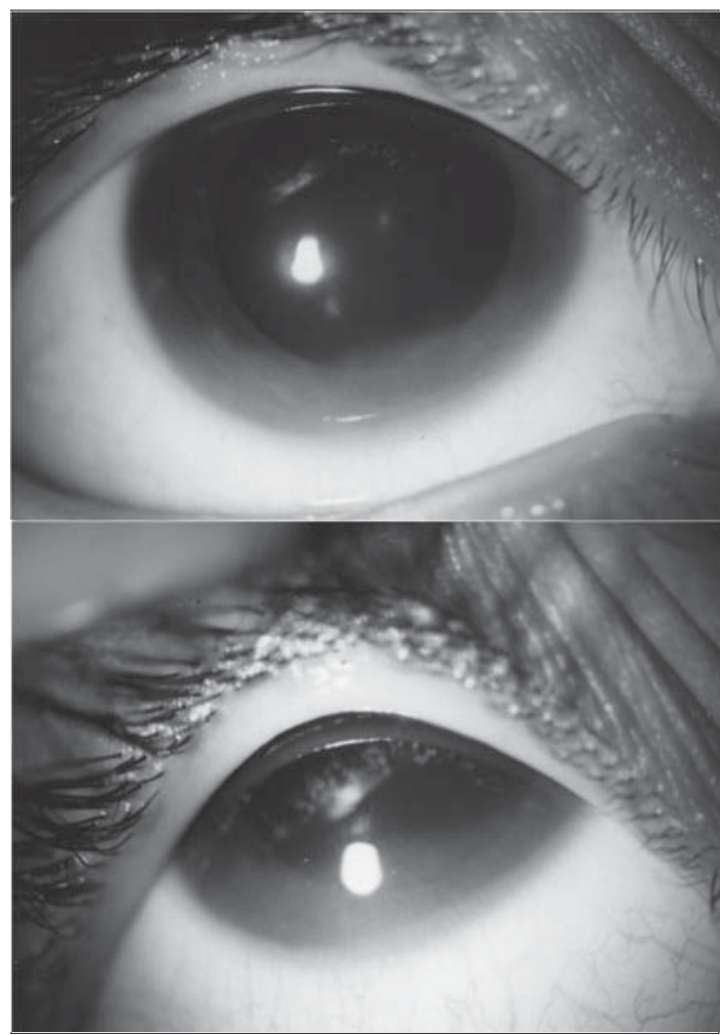

Figura 1. Fotografías de segmento anterior del ojo derecho año 2001 con opacidad corneal inferior y precipitados queráticos finos. estudio de uveítis de origen infeccioso para toxoplasmosis, toxocariasis, sífilis y tuberculosis e interconsulta al reumatólogo infantil para estudio de enfermedad autoinmune sistémica.

En esta última evaluación, no se encontraron antecedentes clínicos de artritis ni signos físicos de conectivopatía. En decisión conjunta

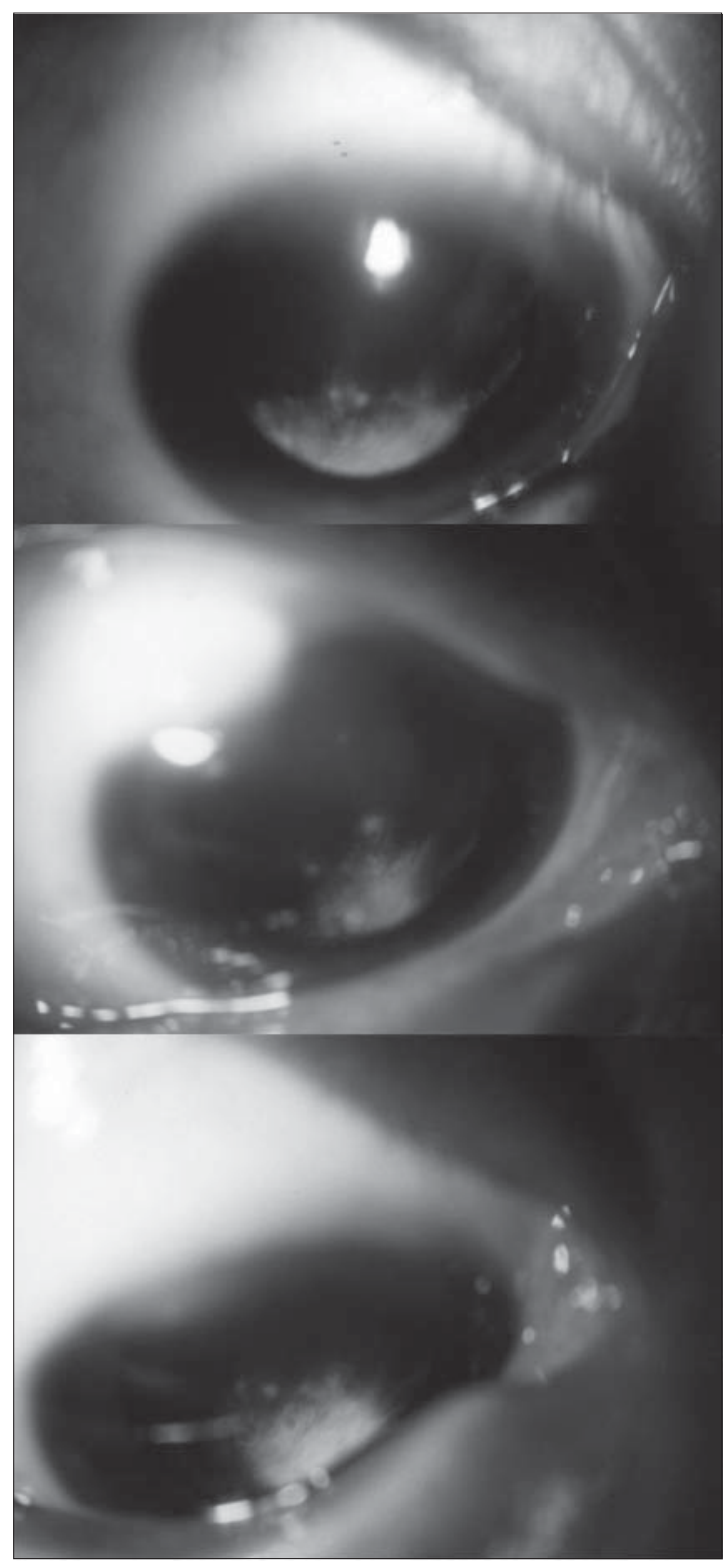

Figura 2. Fotografías clínicas del ojo derecho del año 2001 en infraducción: Nótese la presencia de opacidad blanquecina densa inferior ("snowbank") y opacidades pequeñas esféricas adyacentes sobre el "snowbank" ("snowballs"). 
entre oftalmólogos y reumatólogo infantil, se decidió esperar el resultado de estos exámenes solicitados, los que finalmente resultaron todos con serología negativa para infección.

El estudio inmunológico mostró factor reumatoídeo, anticuerpos antinucleares, complementemia y anticuerpos antiestreptolisina $\mathrm{O}$ en valores normales. P-ANCAS, C-ANCAS y electroforesis de proteínas también resultaron en rangos normales. Sólo inmunoglobulinas séricas resultaron alteradas por discreta elevación de la IgE.

En junio del 2001 se constató empeoramiento del cuadro manifestado en presencia de neovascularización del "snowbank", y disminución de la agudeza visual a 0,12 en su ojo derecho, por lo que se deriva a policlínico de subespecialidad de la Fundación Oftalmológica Los Andes.

Se indicó un examen bajo anestesia general que mostró la presencia de signos de vasculitis retinal periférica inferior y edema papilar, por lo que se efectuó una inyección de fosfato/acetato de betametasona (Cidotén rapilento) $0,8 \mathrm{ml}$.

Un mes después se controló y ante la persistencia de la inflamación se decide una $2^{\mathrm{a}}$ inyección de similares características.

Persiste la enfermedad corneal y Tyndall $(+)$ en cámara anterior en los meses siguientes lo que fue manejado con acetato de prednisolona al 1\%, 1 gota cada 4 horas, luego cada 12 horas hasta octubre 2001. Se solicitó tomografía de coherencia óptica (OCT) de la retina, que confirmó persistencia de edema macular secundario a su uveítis crónica, con un grosor central de $263 \mathrm{um}$. A esta altura se hace necesario el uso de esteroides sistémicos y de acuerdo a sugerencia del reumatólogo infantil se inicia prednisona $30 \mathrm{mg}$ cada 12 horas, isoniacida $150 \mathrm{mg}$ al día, restricción de sodio, suplementación con calcio y vitamina $\mathrm{D}$.

Durante el seguimiento, su agudeza visual mejora a 0,5 en Diciembre del 2001 pero tiende a caer hacia Abril del 2002. Al examen oftalmológico presentaba alteración de la curvatura de la cara posterior del cristalino por la presencia de placa fibrosa del "snowbank", más opacidad subcapsular posterior inicial en el cristalino, provocando un astigmatismo alto y desarrollo de ambliopía por anisometropía con el paso del tiempo. En Agosto del 2002 sufre reactivación del cuadro uveítico, manifestado por ojo rojo y pérdida de visión secundaria al aumento del edema macular del ojo derecho, lo que obliga a aumentar nuevamente dosis sistémicas de corticoides. Debido a que el niño no toleraba disminución bajo $10 \mathrm{mg}$. Al día de Prednisona oral, sin reactivar inflamación intraocular, se agregó metotrexato $12,5 \mathrm{mg}$ a la semana en Agosto del 2003, junto a suplemento de ácido fólico, decisión terapéutica que fue concomitante con la positivización de los anticuerpos antinucleares hasta 1/80 con patrón moteado, $y$ que previamente habían sido negativos. Su agudeza visual mejor corregida en ese entonces era de 0,2 en su ojo enfermo.

A comienzos del 2003 el niño había presentado sintomatología en articulación de la cadera izquierda por lo que se evaluó con clínica y radiología en conjunto con ortopedia infantil. Frente a este debut tardío de artritis, y a la presencia de anticuerpos antinucleares en 1/ 160 hacia septiembre 2005 fue planteándose la hipótesis diagnóstica de vasculitis ocular secundaria a artritis idiopática juvenil, por reumatólogo.

Desde el punto de vista ocular, el paciente se mantuvo con visión corregida en su ojo derecho de 0,12 hasta agosto del 2004 . Nuevo examen de fondo de ojo mostró aspecto aún marcado del "snowbank", y persistencia de engrosamiento macular secundario a edema, por lo que se consideró utilizar una nueva arma terapéutica local a través de una inyección de acetónido de triamcinolona (KENALOG) $4 \mathrm{mg} / 0,1$ $\mathrm{ml}$ intravítreo en ojo derecho, bajo anestesia general, que se realiza en octubre 2004, en concomitancia a su tratamiento con prednisona oral $15 \mathrm{mg}$ día por medio, metotrexato $12,5 \mathrm{mg}$ a la semana oral e isoniacida.

Posterior a esta inyección, el paciente cursa un período de inactividad inflamatoria, con un grosor macular sin gran variación al OCT seriado. A fines del 2005 presentó 2 episodios seguidos de infecciones respiratorias bajas, por lo que también fue estudiado por Inmunología y se cuantificaron Ig séricas resultando normales y anticuerpos antinucleares en 1/80. Su visión corregida fue de 0,12 , la que se mantuvo hasta su último control en Junio del 2007, con examen 
biomicroscópico demostrando una córnea clara, sin opacidad ni engrosamiento, cristalino con catarata subcapsular posterior y vítreo sin actividad inflamatoria.

\section{Discusión}

Estudios en pacientes pediátricos chilenos con uveítis han mostrado que las causas más frecuentes de inflamación intraocular han sido toxoplasmosis, toxocariasis y Pars planitis ${ }^{17}$. En esta población, la Uveitis Intermedia dio razón de un $11,7 \%$ del total de niños con uveítis, siendo todos los casos correspondientes a Pars Planitis y el $100 \%$ de ellas con un curso de carácter crónico ${ }^{18}$. La asociación sistémica más frecuentemente encontrada en niños con uveítis fue artritis reumatoide juvenil.

La PP es un subtipo crónico de UI caracterizado por infiltración celular inflamatoria del vítreo y vasculitis retinal periférica. Puede asociarse o no a inflamación del segmento anterior del ojo de intensidad variable. Se reserva el término PP para aquel subtipo idiopático de uveítis intermedia, en ausencia de infección o enfermedad sistémica ${ }^{6}$.

Hay otras formas de uveitis intermedia que si pueden estar asociadas a enfermedades generales tales como esclerosis múltiple o sarcoidosis, e infecciones de diverso origen como enfermedad de Lyme ${ }^{7}$, toxocariasis, virus HTLV- ${ }^{15}$ o Epstein-Barr ${ }^{7,16}$.

El origen de la PP es aún un enigma, aunque algunos estudios clínicos, inmunogenéticos e histopatológicos han sugerido un proceso autoinmune con predisposición genética ${ }^{19}$. Su incidencia se estima en 1,5 por 100.000 habitantes/ año ${ }^{8}$.

Los pacientes con PP pueden quejarse de entopsias y visión borrosa y típicamente presentan un ojo blanco y tranquilo, incluso diagnosticarse en forma accidental en un examen de rutina. Ojo rojo, dolor, lagrimeo pueden aparecer, pero son menos comunes. Alrededor de un tercio de los pacientes con compromiso unilateral pueden desarrollar compromiso bilateral durante el seguimiento a largo plazo.

En Chile, se ha encontrado que en 277 pacientes de población uveítica en general, un $6,5 \%$ de todas las uveítis correspondían a UI, todas de etiología desconocida, la mayoría unilaterales y con precipitados queráticos finos, sin describir si había o no, presencia de endoteliopatía autoinmune?.

Años atrás, sólo se destacaba la importancia del compromiso del segmento posterior del ojo dentro de la presentación clínica de $\mathrm{UI}^{10}$, hasta que Khodadoust, estableció una asociación entre PP y endoteliopatía corneal como fenómenos autoinmunes relacionados en el ojo ${ }^{4}$.

En un estudio de población mestiza mexicana con PP, el motivo de consulta más frecuente fue la disminución de agudeza visual y en un pequeño grupo de pacientes fue la presencia de una mancha blanca en el ojo afectado $(8,7 \%)$, notado por padres o profesores, tal como sucedió en el caso relatado, y que podía deberse a presencia de queratopatía en banda, endoteliopatía corneal autoinmune, catarata o membrana ciclítica retrolental. Por género, los hombres fueron más afectados que las mujeres, con un $65,6 \%$ de los $\operatorname{casos}^{14}$.

La presentación de endoteliopatía corneal autoinmune y pars planitis es un evento de rara ocurrencia y existen pocos trabajos en la literatura mundial al respecto ${ }^{11}$. Pese a ello, un estudio sobre 166 pacientes pediátricos habría mostrado que hasta un $25 \%$ de ellos presentaban signos de endoteliopatía corneal periférica. No se encontró ninguna relación entre gravedad de la inflamación y presencia de endoteliopatía ${ }^{12}$, pero si se ha reconocido que la opacidad corneal es más frecuente en pacientes de edad pequeña, que en adolescentes o adultos jóvenes ${ }^{13}$.

La endoteliopatía corneal autoinmune es un hallazgo infrecuente en el segmento anterior asociado a PP. Se trata de un edema corneal periférico que se asocia con una línea de precipitados queráticos que separa las áreas de edema de la córnea clara, que requiere una búsqueda dirigida, ya que puede pasarse por alto en un examen oftalmológico poco cuidadoso, $\mathrm{y}$ no efectuar un diagnóstico oportuno de PP, con las consecuencias ya mencionadas.

Dentro de la historia clínica de una UI, es muy importante recoger antecedentes que permitan diferenciar la PP de la UI asociada a enfermedades sistémicas.

A modo de ejemplo, la exposición a garrapatas más antecedentes de rash cutáneo o ar- 
tritis puede sugerir enfermedad de Lyme $^{20}$, mientras que el contacto con gatos puede apuntar al diagnóstico de Bartonella ${ }^{21}$. Otra causa importante de uveítis a descartar es sífilis, solicitando VDRL y pruebas treponémicas. La fiebre, sudoración nocturna y fatiga pueden estar asociados con sarcoidosis o TBC, y para ello deben evaluarse calendario de inmunizaciones, PPD, baciloscopía y radiografía de tórax, según necesidad, ante la posibilidad de uso de inmunosupresión sistémica.

La relación entre esclerosis múltiple y UI ha sido bien documentada y esta puede preceder, ser concomitante o posterior a ella. Usualmente son paciente de género femenino con inflamación ocular bilateral y signos de periflebitis retinal $^{22}$.

No hay examen que confirme el diagnóstico de PP. Su diagnóstico es clínico, basado en la ausencia de compromiso coroídeo, mínimo compromiso de segmento anterior y manifestaciones inflamatorias en el vítreo y retina periférica.

La angiografía retinal con fluoresceína es útil para evaluar las anomalías vasculares de la retina como hiperfluorescencia venosa (tinción parietal), hiperfluorescencia capilar ("patrón en helecho"), hiperfluorescencia de la papila y edema macular ("patrón petaloídeo").

En individuos con opacidad de medios, se solicita una ecografía ocular bidimensional para descartar complicaciones tales como membranas ciclíticas, bandas de tracción vítreas, desprendimiento de retina o hemorragias vítrea.

La PP está asociada a un espectro variado de severidades y curso clínico. Hasta el momento no se han identificado marcadores de laboratorio útiles que predigan la evolución a largo plazo de esta uveítis ${ }^{19}$.

En el tratamiento de la uveítis infantil, se recomienda un tratamiento temprano y agresivo antes que la agudeza visual caiga de 0,5 , muchas veces de forma irrecuperable ${ }^{23}$.

Después de descartar entidades tratables infecciosas y no infecciosas, el primer paso consiste en utilizar corticoides tópicos ante la presencia de inflamación de segmento anterior, junto a corticoides locales subtenonianos y/o intravítreos para las manifestaciones posteriores. El acetónido de triamcinolona intravítreo ha sido efectivo en disminuir rápidamente la inflamación en uveítis no infecciosa con compromiso visual agudo y severo ${ }^{24}$.

Como segundo paso, algo menos utilizados son los antiinflamatorios no esteroidales por vía sistémica ante la recurrencia de la inflamación, después de una serie de inyecciones locales de esteroides, así como en forma de gotas oftálmicas para el manejo del edema macular uveítico.

Si la inflamación persiste, debiera considerarse el uso de corticoides sistémicos orales por un período de evaluación de hasta 3 meses. En el caso que se requiriera mantener estos medicamentos a largo plazo, drogas inmunosupresoras pueden postularse, siendo de $1^{\mathrm{a}}$ línea el metotrexate, ciclosporina y el micofenolato mofetil. También se han utilizado azathioprina ${ }^{25}$ y como última línea, ciclofosfamida ${ }^{12}$ y clorambucil. La vitrectomía es otra alternativa terapéutica ante la refractariedad a los esteroides.

Las complicaciones de la PP en niños incluyen queratopatía en banda, catarata, glaucoma, desprendimiento de retina, hemorragia vítrea y ambliopía sensorial y/o anisometrópica, explicando en parte, esta última causa, la falta de recuperación visual en este niño junto al edema macular crónico.

El compromiso del nervio óptico en UI es observado más comúnmente en niños que en adultos. La neovascularización de retina y nervio óptico puede ser fuente de hemorragia vítrea, principal indicación de cirugía vitreoretinal en UI.

La facoéresis con o sin implante de lente intraocular se ha utilizado en catarata 2aria a inflamación crónica y/o uso de corticoides. La fotocoagulación láser o crioterapia son alternativas en casos de neovascularización del polo posterior $\mathrm{y} / \mathrm{o}$ desgarros retinales.

La alta prevalencia de complicaciones oculares derivadas de la PP en niños, comparados con los adultos, es mejor explicada por el curso más asintomático de la UI en niños, referencia más tardía y mayor grado de daño irreparable al momento de la presentación ${ }^{1}$.

Los fenómenos autoinmunes del ojo pueden asociarse, a su vez, a procesos generales de la misma naturaleza y estos pueden ser contemporáneos ó aparecer más adelante en el tiempo, como el caso presentado, que debutó primero en el ojo y 4 años después de forma sistémica. 
A modo de ejemplo, se ha reportado que glomerulonefritis y uveitis también pueden compartir patogénesis inmunológica común, incluyendo depósito de complejos inmunes circulatorios, entre otros mecanismos ${ }^{26,27}$.

Finalmente, estimamos valioso, el trabajo conjunto entre oftalmólogo y reumatólogo infantil no sólo en el enfrentamiento de esta enfermedad misteriosa y compleja aún, sino también llevando a cabo estudios prospectivos y vigilancia clínica de reumatismos de partes blandas u otras afecciones, con compromiso ocular potencial $^{28,29}$.

\section{Referencias}

1.- Nussenblatt RB, Whitcup SM, Palestine AG: Intermediate Uveitis. En: Craven L, editors. Uveitis, fundamentals and clinical practice. St. Louis: Mosby 1996; 20: 291-300.

2.- De Boer J, Berendschot T, Van der Does P, Rothova A: Long-term follow-up of Intermediate Uveitis in Children. Am J Ophthalmol 2006; 141 (4): 616-21.

3.- Palimeris G, Marcomichelakis N, Konstantinidou V, Trakaniari AN: Intermediate uveitis: what is the natural course of the disease its relationship with other systemic diseases?. Eur J Ophthalmol 1994; 4 (4): 223-7.

4.- Khodadoust AA, Karnama Y, Stoessel KM, Puklin JE: Pars planitis and Autoimmune Endotheliopathy. Am J Ophthalmol 1986; 102 (5): 633-9.

5.- Paul RH: Presumed autoimmune corneal endotheliopathy. Am J Ophthalmol 1988; 105 (5): 519-22.

6.- Jabs DA, Nussenblatt RB, Rosenbaum JT: Standardization of Uveitis Nomenclature (SUN) Working Group. Standardization of uveitis nomenclature for reporting clinical data. Results of the First International Workshop. Am J Ophthalmol 2005; 140: 509-16.

7.- Boskovich SA, Lowder CY, Meisler DM, Gutman FA: Systemic diseases associated with intermediate uveitis. Cleve Clin J Med 1993; 60 (6): 460-5.

8.- Gritz DC, Wong IG: Incidence and prevalence of uveitis in Northern California: the Northern California Epidemiology of Uveitis Study. Ophthalmology 2004; 111: 491-500.

9.- Vidal $R$, Villarroel F, Varela H, et al: Distribución de morbilidad en el Departamento de Uveitis, Servicio de Oftalmología del Hospital del Salvador, 1987-1991. Arch Chil Oftalm 1992; 49 (1): 45-51.

10.- González G: Uveitis intermedia. Cienc Oftalmol 1985; 5: 21-29.

11.- Suazo L: Uveitis intermedia y sus complicaciones. Anales oftalmológicos 2005; 1 (1) : 24-30.

12.- Arellanes-García L, Navarro-López LP, Recillas-
Gispert C: Pars planitis in the Mexican Mestizo population: Ocular findings, treatment, and visual outcome. Ocul Immunol Inflamm 2003; 11 (1): 5360.

13.- Oréfice F, Lima MS, Boratto LM: Uveíte Intermediária (Pars Planite) e Endoteliopatia Corneana Auto Imune (Estudo de 4 casos)-Parte II. Rev Bras Oftalmol 1993; 52 (2): 13-7.

14.- Arellanes-Garcia L, Ortiz-Ponce G, Recillas-Gispert C: Peripheral corneal endotheliopathy and pars planitis. Ocul Immunol Inflamm 1996; 3: 135-8.

15.- Mochizuki M, Watanabe T, Yamaguchi K, et al: Uveitis associated with human T-cell lymphotropic virus type 1. Am J Ophthalmol 1992; 114: 123-9.

16.- Pérez-Álvarez AF, Jiménez-Alonso J, Reche-Molina, et al: Retinal vasculitis and vitreitis in a patient with chronic hepatitis C virus. Arch Intern Med 2001; 161: 2262.

17.- Vega R: Uveítis en la infancia. Arch Chil Oftalm 1995; 52 (1): 41-53.

18.- Vega R: Uveitis in childhood in Santiago, Chile. En: Dodds E. And Couto C. Ed: Uveitis in the Third Millenium, Buenos Aires: Elsevier 2000; 171-4.

19.- Holland G: The enigma of Pars Planitis, revisited. Am J Ophthalmol 2006; 141: 729-30.

20.- Breeveld $J$, Rothova A, Kuiper $H$ : Intermediate uveitis and Lyme borreliosis. Br J Ophthalmol 1992; 76: 1812.

21.- Soheilian M, Markomichelakis N, Foster CS: Intermediate uveitis and retinal vasculitis as manifestations of cat scratch disease. Am J Ophthalmol 1996; 122: 582-4.

22.- Prieto JF, Dios E, Gutiérrez JM, Mayo A, Calonge M, Herreras JM: Pars planitis: epidemiology, treatment, and association with multiple sclerosis. Ocul Immunol Inflamm 2001; 9 (2): 93-102.

23.- Levy-Clarke GA, Nussenblatt RB, Smith JA: Management of chronic pediatric uveitis. Curr Opin Ophthalmol 2005; 16 (5): 281-8.

24.- Benitez del Castillo Sánchez JM, García Sánchez J: Intravitreal injection of triamcinolone acetonide in non infectious uveitis. Arch Soc Esp Ophthalmol 2001; 76 (11): 661-4.

25.- Cuchacovich M, Pacheco P, Díaz G, et al: Role of Azathioprine in steroid resistant non infectious ocular inflammatory diseases. Rev Méd Chile 2007; 135 (6): 702-7.

26.- García de Cortazar L, Alvarado C, Las Heras J: Síndrome nefritis intersticial y uveítis. Rev Chil Pediatr 1991; 62 (4): 252-6.

27.- Román E, Zamora I, Vera F: Mesangial glomerulonephritis and intermediate uveitis. Nefrología 2004; 24 (5): 489-92.

28.- Miranda AM, Talesnik GE, González MB, et al: Enfermedades reumáticas y del tejido conectivo en niños de Santiago, Chile. Rev Chil Pediatr 1996; 67 (5); 200-5.

29.- Morales M: Uveitis infantil. Cienc Oftalmol 2001; 17 (1): 5-9. 